\title{
In-situ EBSD characterization of deformation behavior of primary alpha phase in Ti-6Al-4V
}

Wansong Li, Shigeto Yamasaki, Masatoshi Mitsuhara, Hideharu Nakashima

Department of Advanced Materials Science and Engineering, Kyushu University, Fukuoka 8168580, Japan

Corresponding author: Wansong $\mathrm{Li}$

Email: li.wansong.136@m.kyushu-u.ac.jp

\begin{abstract}
$\underline{\text { Abstract }}$
Uniaxial tension experiments and electron back-scatter diffraction were performed on a bimodal Ti-6Al-4V alloy to study the deformation behavior of primary hep-Ti $\left(\alpha_{\mathrm{p}}\right)$. It was found that the obtained tensile strength and elongation of the studied Ti-6Al-4V from the in-situ tensile test are higher than of which derived from the regular tensile test. The strain could be accommodated by the activation of slip systems and by grain rotations during the deformation. The prismatic slip is the primary slip mode of $\alpha_{\mathrm{p}}$. According to kernel average misorientation analysis, we found that the dislocations mainly distributed near grain boundaries and subgrain boundaries, and partially located around slip lines. Calculated rotation angles and average rotation rates show that the rotation heterogeneity occurred among grains and subgrains.
\end{abstract}

Keywords: Electron backscatter diffraction, Microstructure, Deformation, Grain rotation.

\section{Introduction}

A typical Ti-6Al-4V with bimodal microstructure consists of equiaxed primary hcp-Ti $\left(\alpha_{\mathrm{p}}\right)$ and lamellar secondary alpha $\left(\alpha_{\mathrm{s}}\right) / \mathrm{bcc}-\mathrm{Ti}(\beta)$. Its deformation process mainly depends on the effect of alpha phase due to the low volume fraction of beta phase [1]. $\alpha_{\mathrm{p}}$, being the major constitute (more than $50 \%$ in vol.\%) of all the alpha phase, plays a significant role in resisting the plastic deformation.

Understanding the deformation behavior of $\alpha_{\mathrm{p}}$ is useful to improve the deforming capabilities of the material. Many studies have devoted to the process of strain-induced twinning [2], slip transmission [3,4], active slip system [5,6], and the effect of strain rate $[7,8]$ on the titanium alloys with dual phases using tension or compression experiments. Besides, it was found in the aluminum alloy that grain rotation would undergo under the plastic deformation [9,10]. Such phenomena can also be expected to occur in Ti-6Al-4V.

By previously studies, the deformation mechanism of $\alpha_{\mathrm{p}}$ in the bimodal Ti-6Al-4V alloy is still not completely understood, especially grain rotation behavior of $\alpha_{\mathrm{p}}$ rarely reported. Therefore, the present work aims at investigating the deformation behavior of $\alpha_{p}$ in bimodal Ti-6Al-4V alloy by electron backscatter diffraction (EBSD) and in-situ tensile tests. The slip systems and grain rotation behavior during deformation were discussed.

\section{Experimental}

2.1. Materials

The studied material was an ingot of Ti-6.4Al-4.2V-0.2O-0.2Fe (at.\%) alloy, which was subjected to solution treatment at an intercritical temperature of $940{ }^{\circ} \mathrm{C}$ for $2 \mathrm{~h}$, and subsequently, water-cooled to room temperature-followed by aging heat treatment at $705{ }^{\circ} \mathrm{C}$ for $3 \mathrm{~h}$, and air-cooled to room temperature. All the specimens were cut from the plate after the heat treatment.

2.2. In-situ tensile tests and EBSD measurements

The sample for the in-situ tensile test was prepared using mechanical processing with a gauge length of $6 \mathrm{~mm}$ and a gauge width of $2 \mathrm{~mm}$ and a thickness of $1 \mathrm{~mm}$. Displacement-controlled in-situ tensile tests were performed using a scanning electron microscope equipped with a tensile tester. The extension rate was at $5 \times 10^{-4} / \mathrm{s}$. The insitu tensile test suspended (interrupted) when the displacement was up to the given value. Then, EBSD measurement would be performed with a scanning step size of $0.5 \mu$ m. The corresponding EBSD data were post-processed by OIM 7 software.

2.3. Regular tensile test

The specimen for the regular tensile test was cut into the same shape and tested at the same condition with the sample for the in-situ tensile analysis. While the regular (continuous) tensile test did not suspend until it was broken off.

\section{Results and Discussion}

3.1. Strain-stress curves

Fig. 1 shows the strain-stress curves of tensile tests, in which the black and gray correspond to in-situ tensile and regular tensile test, respectively. As presented in this figure, the in-situ tensile test was interrupted six times to do the in-situ EBSD experiment. It had six small loading drops (numbered 1-6) due to stress relaxation. Table 1 listed the corresponding strains for the six drops. Mechanical properties of the specimen for the in-situ and regular tensile test was presented in Table 2 . Table 2 indicates that there was almost no difference in the yield strength of the two samples obtained from the in-situ and regular tensile tests. However, the tensile strength and elongation of the specimen obtained by the in-situ tensile test were higher than of the sample obtained by regular tensile test. This phenomenon may be caused by the creep deformation that happened during the loading stops of the in-situ tensile test [11]

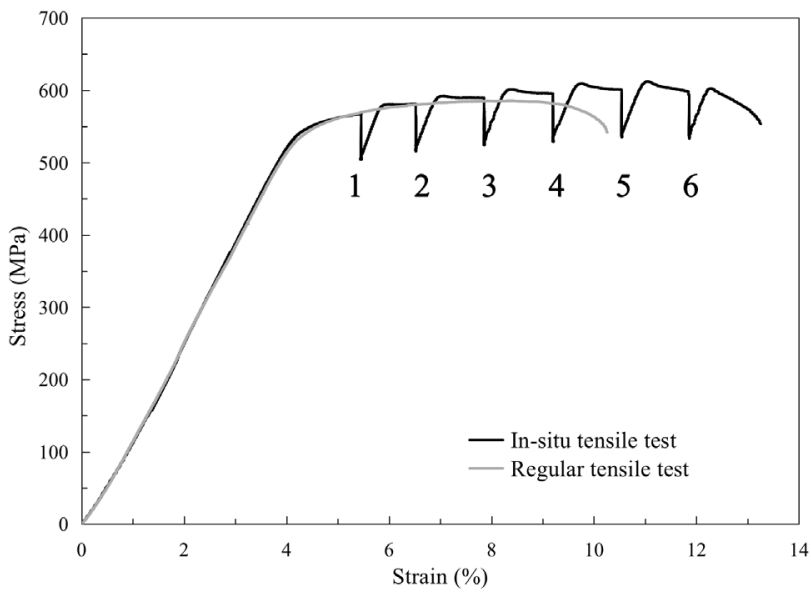

Fig. 1. Stress-strain curves of the specimen recorded during the in-situ (black) and regular tensile test (gray).

(C) The Authors, published by EDP Sciences. This is an open access article distributed under the terms of the Creative Commons Attribution License 4.0 (http://creativecommons.org/licenses/by/4.0/). 
Table 1. Drops and corresponding strains obtained from the in-situ tensile test for the Ti-6Al-4V.

\begin{tabular}{lllllll}
\hline Drop & 1 & 2 & 3 & 4 & 5 & 5 \\
\hline Strain $(\%)$ & 5.5 & 6.5 & 7.9 & 9.2 & 11.9 & 10.5 \\
\hline
\end{tabular}

Table 2. Mechanical properties of two Ti-6Al-4V specimens measured from the in-situ and regular tensile tests.

\begin{tabular}{lllll}
\hline Specimen & Yield strength $(\mathrm{MPa})$ & Tensile strength $(\mathrm{MPa})$ & Uniform elongation $(\%)$ & Total elongation $(\%)$ \\
\hline In-situ & 541 & 612 & 11.0 & 13.3 \\
Regular & 536 & 586 & 8.4 & 10.3 \\
\hline
\end{tabular}

3.2. Microstructure

Fig. 2(a) shows the secondary electron (SE) morphology of the specimen before loading the strain. This figure shows that the specimen had a typical bimodal structure consisting of $\alpha_{\mathrm{p}}$ and lamellar $\alpha_{\mathrm{s}} / \beta$, and was also used to distinguish $\alpha_{\mathrm{p}}$ and $\alpha_{\mathrm{s}}$ from the EBSD maps. Fig. 2(b) shows the inverse pole figure (IPF) images of the microstructure, corresponding to the same area in Fig. 2(a). Fig. 2(c), (d), and (e) show the IPF maps of $\beta, \alpha_{\mathrm{p}}$, and $\alpha_{\mathrm{s}}$, respectively. Three axes of the specimens were defined in Fig. 2. Namely, the view direction of the figure is for the normal direction (ND) of the plate specimen, the horizontal direction for the loading direction (LD), and the vertical direction for the transverse direction (TD). As presented in Fig. 2(c), the $\beta$ phase was homogeneously distributed in the matrix. However, it only took up 2.3\% (vol.) in the microstructure. As displayed in Fig. 2(d) and (e), the $\alpha_{\mathrm{p}}$ and $\alpha_{\mathrm{s}}$ were able to be separated from the IPF maps by their different morphology feature obtained from the SE image (Fig. 2(a)). The ratio of $\alpha_{\mathrm{p}}$ was about $56.8 \%$ (vol.) in the microstructure.
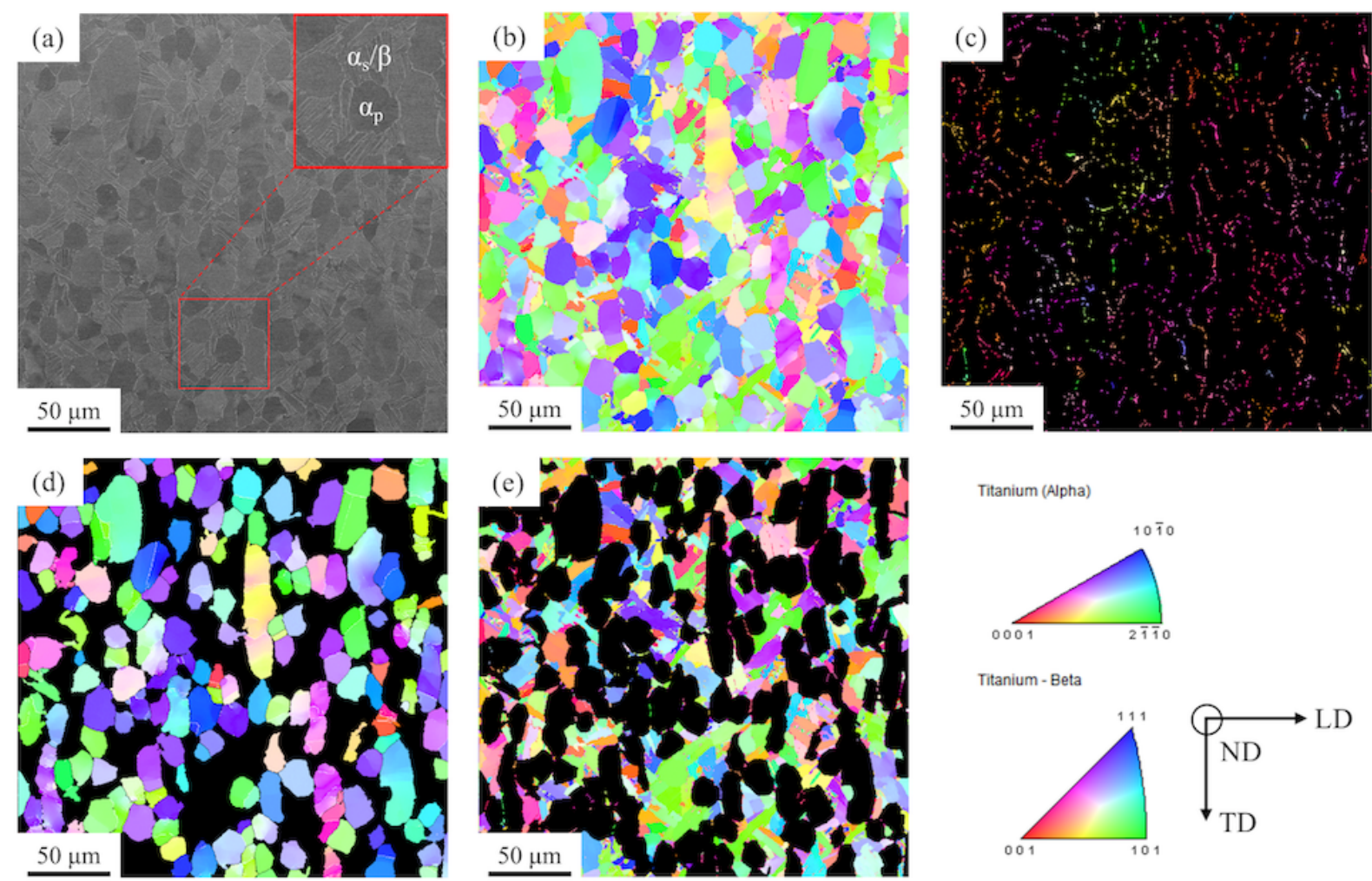

Titanium - Beta

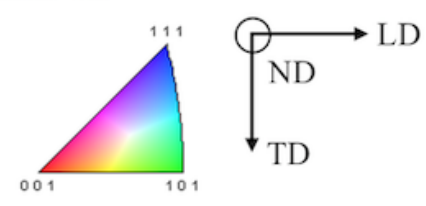

Fig. 2. Microstructures of the specimen for the same area before loading the strain:(a) secondary electron morphology, (b) IPF of the specimen, (c) IPF of $\beta$ phase, (d) IPF of $\alpha_{p}$, (e) IPF of $\alpha_{\mathrm{s}}$. (ND: normal direction, TD: transverse direction, LD: loading direction)

\subsection{Activated slip system}

Slip systems of grains were activated to accommodate the strain. We constructed the plane trace analysis to confirm the specific activated slip system of each grain, as displayed in image quality (IQ) of Fig. 3(a) that corresponds to the same area in Fig. 2(d) at a strain of 5.5\%. Table 3 listed the statistical slip systems of 102 grains. It could be found that the prismatic slips were mainly $(66.7 \%)$ activated during the strain. In the figure of Fig. 3(b), the typical slip lines as a successive straight and regularly spaced morphology exist in $\alpha$ phase. Furthermore, the slip lines of basal are finer than of prismatic and $1^{\text {st }}$-order pyramidal slip lines existing in the microstructure. Such different morphology in slip lines with different activated slip systems was also discovered in a polycrystalline Co-Cr-Mo alloy [12]. 


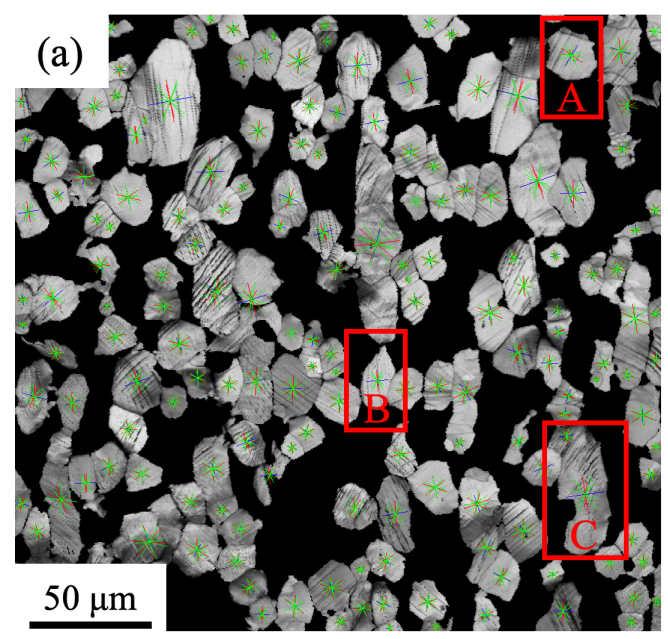

(b)
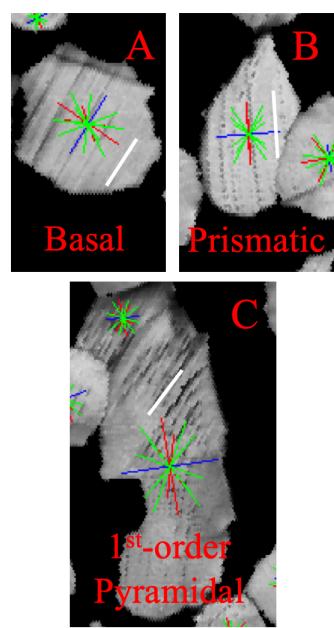

Fig. 3. (a) Image quality of $\alpha_{p}$ after $5.5 \%$ deformation, the blue line traced basal plane, the red line traced prismatic plane, and a green line traced $1^{\text {st }}$-order pyramidal plane, (b) amplified maps of selected areas in (a).

Table 3. The result of the activated slip system in $\alpha_{p}$ after $5.5 \%$ strain in 102 grains.

\begin{tabular}{llllll}
\hline Slip system & Basal & Prismatic & $1^{\text {st }}$-order pyramidal & $2^{\text {nd }}$-order pyramidal & No slip \\
\hline Grain numbers & 17 & 68 & 10 & 0 & 7 \\
\hline
\end{tabular}

3.4. Grain rotation

Grain rotation behavior had found during the strain. As indexed by the red ellipses in IPF maps of Fig. 4, three typical grains (named A1, A2, and A3) were used to characterize the grain rotation. When the strain was up to $5.5 \%$ (Fig.4(b)), grains A1-A3 changed in color of IPF maps but with no transformation in morphology. This change in colors means the change in crystallographic orientation. In other word, this change indicated that these three grains had rotated. When the specimen strained to $7.9 \%$ in Fig. 4(c), grains A1-A3 underwent rotation further. According to the Euler angles defined by the Bunge notation [13], each grain has a specific Euler angle under different strains. From the change of Euler angle of each grain, it was found that the rotation behavior occurred in most ( 92 out of 102 grains) of $\alpha_{p}$ grains in varying degree after $7.9 \%$ strain.
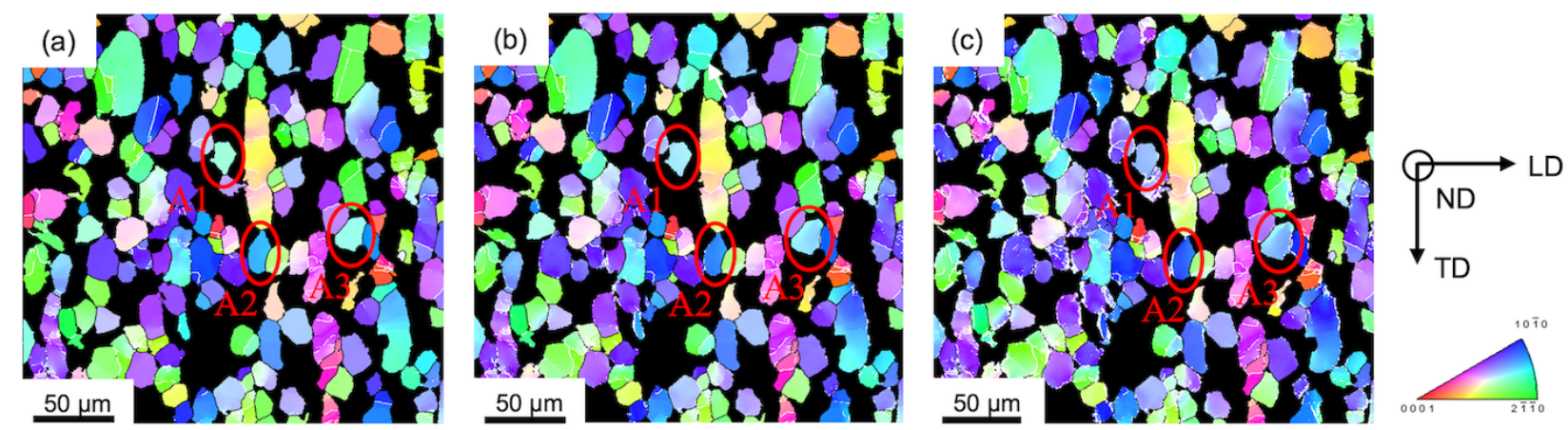

Fig. 4. Inverse pole figures of $\alpha_{p}$ with different true strains: (a) $0 \%$, (b) $5.5 \%$, and (c) $7.9 \%$, respectively.

To further quantify the grain rotations, we selected 12 grains (shown in Fig. 5) and calculated their rotation angles based on the Euler angles of each grain [13]. Table 4 lists the relative rotation angles (degree) during deformation and the average rotation rate $(\% / 1 \%)$ after $10.5 \%$ strain. As listed in Table 4 , it has several rotation heterogeneities in these grains during the deformation. Firstly, the rotation heterogeneity was found within an individual grain after the same strain. For example, the rotation angle of G7 was $1.5^{\circ}$ with the strain varying from $7.9 \%$ to $9.2 \%$ (1.3\% strain difference) and $2.1^{\circ}$ with the strain ranging from $9.2 \%$ to $10.5 \%(1.3 \%$ strain difference). Secondly, the rotation heterogeneity occurred among these grains activated in the same slip mode. For example, G1, G3, and G6 were all activated in basal. However, their average rotation rates are $0.9^{\circ} / 1 \%, 0.7^{\circ} / 1 \%$, and $1.2 \%$, respectively. Such heterogeneity also happened in grains activated in Prismatic and $1^{\text {st }}$-order pyramidal. Thirdly, grains with different active slip modes also show the rotation heterogeneity. For instance, G1 (basal slip), G5 (prismatic slip), and G7 (1 $1_{\text {st }}$-order pyramidal slip) were with the average rotation rate of $0.9^{\circ} / 1 \%, 1.2 \%$, and $1.5^{\circ} / 1 \%$, respectively. Fourthly, the grains with a similar original orientation, exhibit the grain rotation heterogeneity, such as G7 and G9 with the average rotation rate of $1.5 \% 1 \%$ and $0.5 \% 1 \%$, respectively. Such difference also appeared between G2 and G10. Fifthly, subgrains of the grain also show the different rotation rate, which can be proved from the subgrains G2-1, G2-2, and G2-3. As described above, we can infer that the grain rotation heterogeneity strongly depends on the initial orientation of each grain. 


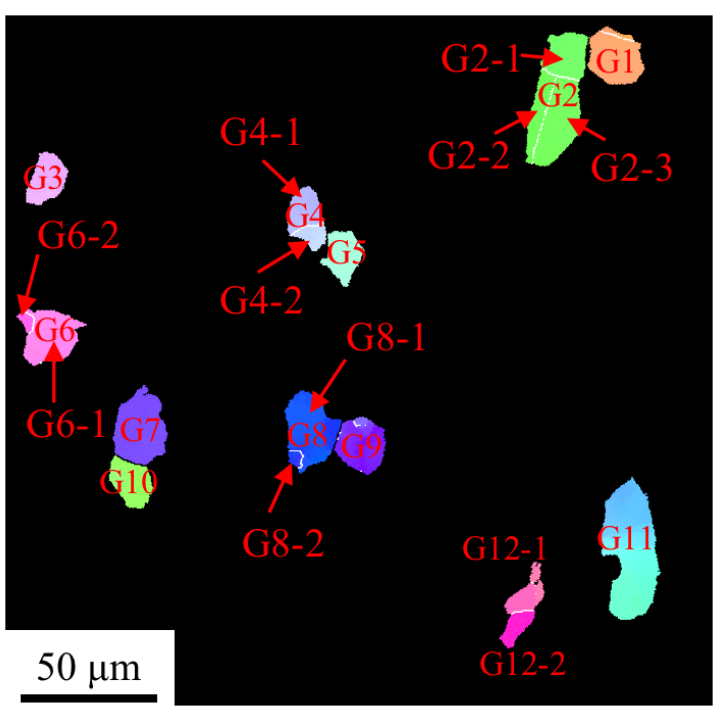

Fig. 5. Selected 12 grains of $\alpha_{p}$ with no strain.

Table 4. Calculated rotation angles and the average rotation rates for selected 12 grains.

\begin{tabular}{|c|c|c|c|c|c|c|c|c|}
\hline \multirow[t]{2}{*}{ Grain } & \multirow[t]{2}{*}{ Subgrain } & \multicolumn{5}{|c|}{ Rotation degree $\left({ }^{\circ}\right)$} & \multirow{2}{*}{$\begin{array}{l}\text { Average rotation } \\
\text { rate }(\% / 1 \%)\end{array}$} & \multirow[t]{2}{*}{ Slip system } \\
\hline & & $\begin{array}{l}\text { From } \varepsilon=0 \% \text { to } \\
\varepsilon=5.5 \%\end{array}$ & $\begin{array}{l}\text { From } \varepsilon=5.5 \% \\
\text { to } \varepsilon=6.5 \%\end{array}$ & $\begin{array}{l}\text { From } \varepsilon=6.5 \% \\
\text { to } \varepsilon=7.9 \%\end{array}$ & $\begin{array}{l}\text { From } \varepsilon=7.9 \% \\
\text { to } \varepsilon=9.2 \%\end{array}$ & $\begin{array}{l}\text { From } \varepsilon=9.2 \% \\
\text { to } \varepsilon=10.5 \%\end{array}$ & & \\
\hline G1 & & 3.6 & 1.0 & 0.7 & 1.2 & 2.9 & 0.9 & Basal \\
\hline \multirow[t]{3}{*}{ G2 } & G2-1 & 5.5 & 3.1 & 6.6 & 0.5 & 3.8 & 1.9 & Prism \\
\hline & G2-2 & 2.1 & 2.9 & 3.4 & 1.6 & 1.9 & 1.1 & Prism \\
\hline & G2-3 & 2.5 & 2.9 & 4.7 & 1.0 & 2.5 & 1.3 & Prism \\
\hline G3 & & 0.7 & 2.7 & 1.4 & 2.0 & 0.9 & 0.7 & Basal \\
\hline \multirow[t]{2}{*}{ G4 } & G4-1 & 2.9 & 3.1 & 5.2 & 1.5 & 3.4 & 1.6 & $1^{\text {st }}$-order Pyramdial \\
\hline & G4-2 & 3.3 & 2.1 & 4.2 & 0.9 & 3.1 & 1.3 & $1^{\text {st }}$-order Pyramdial \\
\hline G5 & & 2.3 & 3.3 & 3.9 & 1.0 & 2.5 & 1.2 & Prism \\
\hline \multirow[t]{2}{*}{ G6 } & G6-1 & 1.9 & 5.8 & 1.5 & 3.3 & 0.5 & 1.2 & Basal \\
\hline & G6-2 & 1.1 & 4.3 & 1.8 & 3.7 & 1.2 & 1.2 & Basal \\
\hline \multirow[t]{2}{*}{ G8 } & G8-1 & 2.1 & 1.2 & 1.0 & 0.5 & 0.1 & 0.5 & Prism \\
\hline & G8-2 & 0.6 & 2.0 & 2.0 & 0.3 & 0.6 & 0.5 & Prism \\
\hline G9 & & 1.2 & 1.6 & 1.2 & 0.6 & 0.5 & 0.5 & Prism \\
\hline G10 & & 0.9 & 1.7 & 0.8 & 1.6 & 0.3 & 0.6 & Prism \\
\hline
\end{tabular}




\begin{tabular}{|c|c|c|c|c|c|c|c|c|}
\hline G11 & & 0.8 & 4.1 & 3.8 & 2.7 & 1.6 & 1.2 & $1^{\text {st }}$-order Pyramdial \\
\hline G12 & G12-1 & 0.6 & 2.5 & 1.3 & 2.0 & 0.7 & 0.7 & Basal \\
\hline & G12-2 & 0.5 & 2.8 & 1.3 & 2.0 & 0.4 & 0.7 & Basal \\
\hline
\end{tabular}

\subsection{Dislocations}

Kernel average misorientation (KAM) is commonly used to analyze the dislocation density of the material quantifiably. Fig. 6(a)-(c) show KAM distribution maps of the same area in Fig. 2(d) at varying strains by imposing a maximum misorientation of $5^{\circ}$ for the nearest neighbor of each point. KAM values (degree) were divided into ranges and displayed as different colors, with blue showing the lowest KAM value $\left(0-1^{\circ}\right)$ and red showing the highest value $\left(4-5^{\circ}\right)$. The misorientations greater than $5^{\circ}$ were excluded from the kernel. As shown that the green and yellow areas of some grains increased continuously with the increasing strain, indicating that KAM values of these grains increased gradually. Also, from these maps that KAM values show different variations within each grain, which proves that the distribution of dislocations is inhomogeneity during the strain in individual grains. In comparison with the IPF maps in Fig. 4, it could be discovered that the dislocations preferentially distributed near grain boundaries and subgrain boundaries. Besides, through a comparison with IQ maps, it is evident in Fig.6(b) that the dislocations also partially concentrated at slip lines, as marked by red arrows at the same area of the grain interior. The average KAM values were calculated to quantify the relationship with strains in the $\alpha_{\mathrm{p}}$, as presented in Fig. 7. As illustrated in this figure, the average KAM value increased with the increase of strain. The evolution of the average KAM value could be attributed to the effect of the strain. The deformation caused slip lines formed in grains and the dislocation glide, then led to a higher strain concentration at the slip line, grain boundaries, and subgrain boundaries. Finally, causing the increase of the local misorientation in these positions with increasing strain [14].
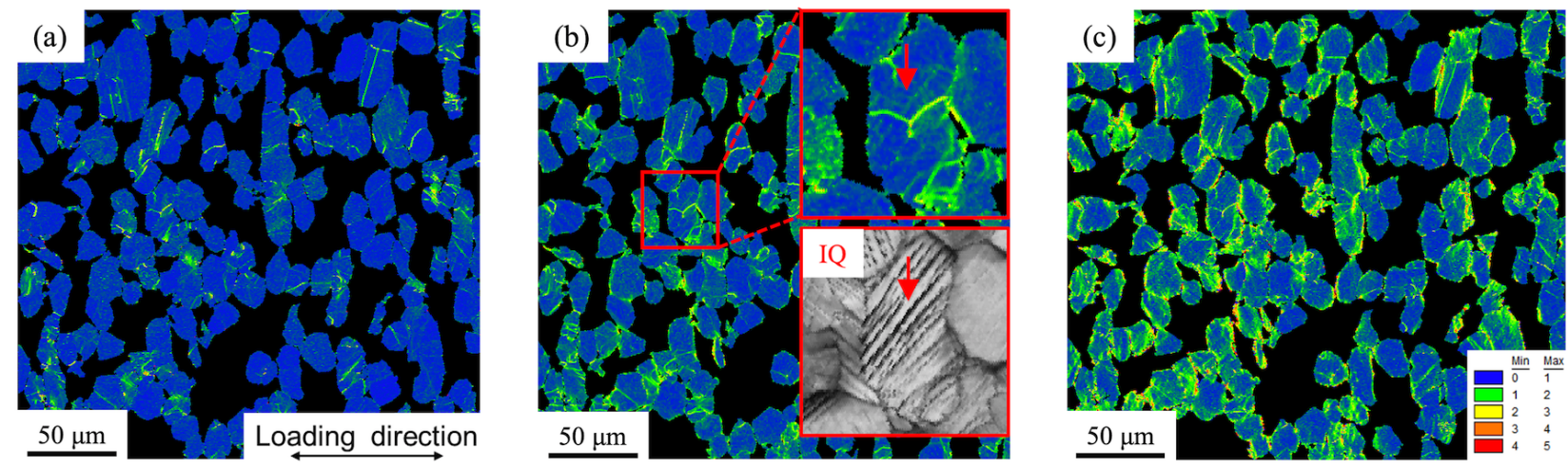

Fig. 6. Color-coded kernel average misorientation map of $\alpha_{p}$ with different true strains: (a) $0 \%$, (b) $5.5 \%$, and (c) $9.2 \%$, respectively.

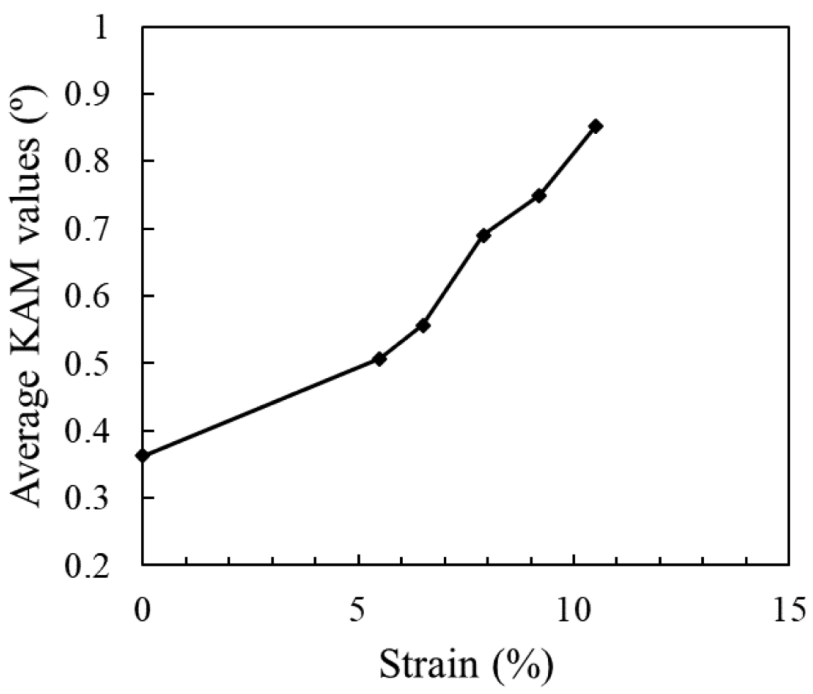

Fig. 7. KAM values with different strains.

\section{Conclusions}

A combination of in-situ tensile test and EBSD technique was used to analyze the deformation behavior of $\alpha_{\mathrm{p}}$ in Ti-6Al-4V. The following conclusions were drawn:

(1) The tensile strength and elongation of the studied Ti-6Al-4V obtained from the in-situ tensile test are higher than of which derived from the regular tensile test.

(2) Dring the in-situ tensile test, basal, prismatic, and 1st-order pyramidal slip systems were activated. The prismatic slip occupied most (66.7\%) of the slip systems.

(3) From the analysis of KAM, the dislocations of $\alpha_{p}$ were majorly concentrated near grain boundaries and subgrain boundaries and partially distributed at slip lines. The average KAM values increased gradually with the increase of the strain.

(4) On terms of the grain rotation angle, and average rotation rate, the rotation heterogeneity occurred among grains and subgrains. Such heterogeneity may be attributed to the initial orientation of each grain.

\section{Acknowledgment}

This research was supported by the Cross-Ministerial Strategic Innovation Promotion Program on Structural Materials for Innovation (SIP-SMI). 


\section{References}

[1] Q. Wang, Z. Liu, Mater. Sci. Eng. A. 675 (2016) 271-279.

[2] Y.G. Liu, M.Q. Li, Mater. Lett. 180 (2016) 47-50.

[3] C. Tan, Q. Sun, L. Xiao, Y. Zhao, J. Sun, J. Alloys Compd. 724 (2017) 112-120.

[4] Z. Zheng, S. Waheed, D.S. Balint, F.P.E. Dunne, Int. J. Plast. 104 (2018) 23-38.

[5] S. Zhang, W. Zeng, Q. Zhao, L. Ge, M. Zhang, Mater. Sci. Eng. A. 708 (2017) 574-581.

[6] F. Bridier, P. Villechaise, J. Mendez, Acta Mater. 53 (2005) 555-567.

[7] Q. Wang, Z. Liu, B. Wang, A.U. Hassan Mohsan, Mater. Sci. Eng. A. 690 (2017) 32-36.

[8] S. Waheed, Z. Zheng, D.S. Balint, F.P.E. Dunne, Acta Mater. 162 (2019) 136-148.

[9] D. Raabe, Z. Zhao, W. Mao, Acta Mater. 50 (2002) 4379-4394.

[10] P. Chen, S.C. Mao, Y. Liu, F. Wang, Y.F. Zhang, Z. Zhang, X.D. Han, Mater. Sci. Eng. A. 580 (2013) 114-124

[11] I.G. Dastidar, V. Khademi, T.R. Bieler, A.L. Pilchak, M.A. Crimp, C.J. Boehlert, Mater. Sci. Eng. A. 636 (2015) 289-300

[12] H. Matsumoto, Y. Koizumi, T. Ohashi, B.S. Lee, Y. Li, A. Chiba, Acta Mater. 64 (2014) 1-11.

[13] V. Randle, O. Engler, Second ed. CRC Press, Boca Raton, FL, (2010) 34-43.

[14] X. Wu, N. Tao, Y. Hong, G. Liu, B. Xu, J. Lu, K. Lu, Acta Mater. 53 (2005) 681-691. 